Check for updates

Cite this: RSC Adv., 2019, 9, 32977

Received 6th August 2019

Accepted 26th September 2019

DOI: 10.1039/c9ra06113d

rsc.li/rsc-advances

\section{Gram-scale synthesis of nitrogen doped graphene quantum dots for sensitive detection of mercury ions and L-cysteine $\uparrow$}

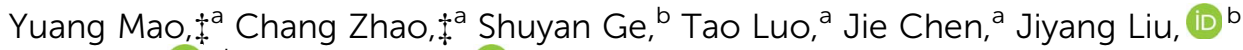 \\ Fengna $X i{ }^{*}{ }^{* b}$ and Junjie Liu (iD *a
}

\begin{abstract}
Sensitive and reliable detection of mercury ions $\left(\mathrm{Hg}^{2+}\right)$ and L-cysteine (L-Cys) is of great significance for toxicology assessment, environmental protection, food analysis and human health. Herein, we present gram-scale synthesis of nitrogen doped graphene quantum dots (N-GQDs) for sensitive detection of $\mathrm{Hg}^{2+}$ and L-Cys. The N-GQDs are one-step synthesized using bottom-up molecular fusion in a hydrothermal process with gram-scale yield at a single run. N-GQDs exhibit good structural characteristics including uniform size $(\sim 2.1 \mathrm{~nm})$, high crystallinity, and single-layered graphene thickness. Successful doping of $\mathrm{N}$ atom enables bright blue fluorescence (absolute photoluminescence quantum yield of $24.8 \%$ ) and provides unique selectivity towards $\mathrm{Hg}^{2+}$. Based on the fluorescence quenching by $\mathrm{Hg}^{2+}$ (turn-off mode), $\mathrm{N}-\mathrm{GQDS}$ are able to serve as an effective fluorescent probe for sensitive detection of $\mathrm{Hg}^{2+}$ with low limit of detection $(19 \mathrm{nM})$. As L-Cys could recover the fluorescence of $\mathrm{N}$-GQDs quenched by $\mathrm{Hg}^{2+}$, fluorescent detection of L-Cys is also demonstrated using turn-off-on mode.
\end{abstract}

\section{Introduction}

Metal ions and small biomarkers play crucial roles in various physiological and pathological processes. ${ }^{\mathbf{1 , 2}}$ For instance, mercury ions $\left(\mathrm{Hg}^{2+}\right)$ are one of the most toxic pollutants and are a great threat to the environment and human health. Even a small intake of $\mathrm{Hg}^{2+}$ ( $2 \mathrm{mg} \mathrm{kg} \mathrm{kg}^{-1}$ body weight per day) can damage the nervous, endocrine and other systems, which further leads to many fatal diseases (e.g. headaches, renal failure, loss of intelligence quotient). ${ }^{3-5}$ On the other hand, as one of the essential amino acids and small biomarkers in human body, L-cysteine (L-Cys) possesses vital physiological functions in neuronal tissues, metabolism and detoxification. ${ }^{6-10}$ Abnormal levels of $\mathrm{L}_{\text {-Cys }}$ can cause growth retardation, neurotoxic effect, Alzheimer's disease and coronary heart disease. ${ }^{8-10}$ Therefore, simple and sensitive detection of $\mathrm{Hg}^{2+}$ and L-Cys is of great importance.

Up to now, many methods including atomic absorption spectrometry (AAS), atomic fluorescence spectrometry (AFS), inductively-coupled plasma mass spectrometry (ICP-MS) and surface-enhanced Raman scattering (SERS) have been

${ }^{a}$ Affiliated Tumor Hospital of Guangxi Medical University, 71 Hedi Road, Nanning 530021, PR China. E-mail: liujunjie186@163.com

${ }^{b}$ Department of Chemistry, Zhejiang Sci-Tech University, 928 Second Avenue, Xiasha Higher Education Zone, Hangzhou, 310018, PR China. E-mail: fengnaxi@zstu.edu.cn $\uparrow$ Electronic supplementary information (ESI) available. See DOI: 10.1039/c9ra06113d

\$ The two authors contributed equally. developed for the detection of $\mathrm{Hg}^{2+} \cdot \mathbf{1 , 1 1 , 1 2}$ However, these methods often require highly extensive instruments and sophisticated operation techniques. Several strategies including high performance liquid chromatography (HPLC) and capillary electrophoresis (CE) have been designed to detect L-Cys. ${ }^{7,10}$ Tedious and time-consuming procedures are usually required. Among various detection techniques, fluorescence detections have attracted considerable interests owing to the ease of operation, rapid detection, high sensitivity, and possibility for real-time monitoring and local observation. ${ }^{\mathbf{8 , 9 , 1 3 , 1 4}}$ Until now, considerable efforts have been devoted to the development of fluorescent probes for monitoring $\mathrm{Hg}^{2+}$ or L-Cys.

Graphene quantum dots (GQDs) or 0D graphene are the newest addition to the nanocarbon family. ${ }^{15-17}$ Owing to its atomic thickness, nanometer scale $(<10 \mathrm{~nm})$ and quantum confinement effect, GQDs exhibit tunable photoluminescence, high photostability, low cytotoxicity, and superior water dispersibility. ${ }^{18,19}$ These unique properties promise a wide range of applications in biological imaging, ${ }^{\mathbf{1 5 , 2 0}}$ catalysis, $^{\mathbf{2 1 - 2 4}} \mathrm{drug}$ delivery, ${ }^{19}$ energy conversion and storage ${ }^{22,25}$ and especially sensing. ${ }^{21,26-32}$ In recent years, it has been proved that heteroatom doping can effectively regulate the property and selectivity of GQDs. Challenge remains in facile and gram-scale synthesis of heteroatom-doped GQDs with specific selectivity.

In this work, we demonstrate gram-scale synthesis of nitrogen doped graphene quantum dots (N-GQDs) for sensitive detection of $\mathrm{Hg}^{2+}$ and L-Cys. As illustrated in Fig. 1, N-GQDs were easily synthesized through one-step bottom-up strategy under hydrothermal condition. The as-prepared N-GQDs show 


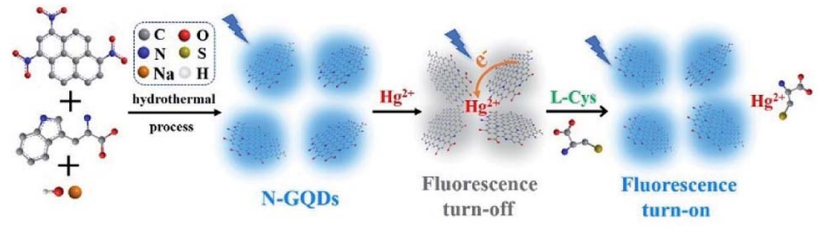

Fig. 1 Schematic illustration for the preparation of N-GQDs and detection of $\mathrm{Hg}^{2+}$ and L-Cys.

bright and emission-independent fluorescence, uniform size, and good crystalline. Owing to specific electron transfer and aggregation, N-GQDs exhibit selective and sensitive fluorescence quenching towards $\mathrm{Hg}^{2+}$. In presence of ${ }_{\mathrm{L}-\mathrm{Cys}}$, the quenched fluorescence of $\mathrm{N}-\mathrm{GQDS} / \mathrm{Hg}^{2+}$ system could be recovered. Based on these fluorescence quenching (turn-off) and recovery (turn-off-on) processes, sensitive and simultaneous detection of $\mathrm{Hg}^{2+}$ and L-Cys are demonstrated.

\section{Experimental}

\subsection{Materials and reagents}

Pyrene, 4-(2-hydroxyethyl)-1-piperazineethanesulfonic acid (HEPES), NaOH, L-cysteine (L-Cys), L-histidine (His), D-methionine (Met), L-tryptophan, L-tyrosine (Tyr), L-leucine (Leu), Lthreonine (Thr), L-alanine (Ala), L-glutamic acid (Glu), L-aspartic acid (Asp) and glycine (Gly) were obtained from Aladdin Chemistry Co. Ltd. (China). The standard solution of $\mathrm{Hg}^{2+}$ was purchased from National Standard Materials Center (China). Aqueous solutions of other cations were prepared from chloride salts $\left(\mathrm{Ni}^{2+}, \mathrm{K}^{+}, \mathrm{Na}^{+}, \mathrm{Ca}^{2+}\right)$, nitrate salts $\left(\mathrm{Zn}^{2+}, \mathrm{Cr}^{3+}, \mathrm{Ag}^{+}, \mathrm{Co}^{2+}\right.$, $\left.\mathrm{Cd}^{2+}, \mathrm{Pb}^{2+}, \mathrm{Fe}^{3+}\right)$, and sulfate salts $\left(\mathrm{Mg}^{2+}, \mathrm{Cu}^{2+}, \mathrm{Al}^{3+}\right)$, respectively. All reagents are of analytical grade and used with further treatment. All aqueous solutions were prepared with ultrapure water (18.2 M $\Omega \mathrm{cm}$, Milli-Q, Millipore).

\subsection{Characterizations}

Transmission electron microscopic (TEM) images was taken on a JEM-2100 transmission electron microscope at operating voltage of $200 \mathrm{kV}$ (JEOL Ltd., Japan). Atomic force microscopic (AFM) images were obtained using tapping mode by Bruker Multimode 8 (Bruker. Inc, USA). Elemental analysis of the NGQDs was performed by X-ray photoelectron spectroscopy (XPS) with PHI5300 electron spectrometer (PE Ltd., USA) using $\mathrm{Mg} \mathrm{K} \alpha$ radiation $(250 \mathrm{~W}, 14 \mathrm{kV})$. Fluorescence spectra were recorded on RF-5301 PC spectrofluorometer (Shimadzu Corporation, Japan). Absolute photoluminescence quantum yield was measured using FL 3C-11 spectrofluorometer (Hariba Scientific, USA).

\subsection{One-step preparation of N-GQDs}

The precursor, 1,3,6-trinitropyrene, was synthesized according to the literature. ${ }^{17}$ N-GQDs were prepared using one-pot hydrothermal process. Briefly, 1,3,6-trinitropyrene (6 mM) and L-tryptophan (75 mM) were dissolved in $\mathrm{NaOH}(125 \mathrm{mM})$ with ultrasonic treatment for $0.5 \mathrm{~h}$. The mixture was then transferred into $500 \mathrm{~mL}$ Teflon-lined autoclave and heated for $6 \mathrm{~h}$ at $200{ }^{\circ} \mathrm{C}$. To optimize the preparation conditions, different concentrations of L-tryptophan (25-120 mM) and $\mathrm{NaOH}(5 \mathrm{mM}$ to $0.5 \mathrm{M})$, reaction temperature $\left(120-200{ }^{\circ} \mathrm{C}\right)$ and time $(2-10 \mathrm{~h})$ were used. After the reaction, the obtained solution was filtered through a $0.22 \mu \mathrm{m}$ microporous membrane to remove insoluble carbon product. N-GQDs could be obtained by freeze-drying after dialysis (retained molecular weight of $1000 \mathrm{Da}$ ) for $24 \mathrm{~h}$ to remove unreacted small molecules. The synthesis yield for $\mathrm{N}$ GQDs was calculated based on the precursor.

\subsection{Fluorescent turn-off detection of $\mathbf{H g}^{2+}$}

The HEPES buffer solution (0.1 M, pH 6.0) containing tartaric acid (TA, $125 \mu \mathrm{M}$ ) was used for the detection of $\mathrm{Hg}^{2+}$. N-GQDs $\left(0.07 \mathrm{mg} \mathrm{mL}^{-1}\right)$ were mixed with $\mathrm{Hg}^{2+}$ at different concentrations $(0.05-25 \mu \mathrm{M})$. After incubation at room temperature for $5 \mathrm{~min}$, the fluorescence (FL) intensity in the absence $\left(F_{0}\right)$ and presence $(F)$ of $\mathrm{Hg}^{2+}$ was measured with the excitation wavelength fixed at $370 \mathrm{~nm}$. The relative fluorescence ratio $\left(F / F_{0}\right)$ and fluorescence quenching ratio $\left(F_{0}-F\right) / F_{0}$ were used for calibration. Each detection was performed in triplicate.

For real sample analysis, the environmental sample was collected from Jinsha Lake (Hangzhou, China). Bacteria, algae and insoluble substances were removed by a $0.22 \mu \mathrm{m}$ microporous membrane. The detection of $\mathrm{Hg}^{2+}$ was evaluated by standard addition method.

\subsection{Fluorescence turn-off-on detection of $\mathrm{L}$-Cys}

As L-Cys can recover the fluorescence of N-GQDs quenched by $\mathrm{Hg}^{2+}$, fluorescence turn-off-on mode was applied to detect L-Cys. Briefly, N-GQDs $\left(0.07 \mathrm{mg} \mathrm{mL}{ }^{-1}\right)$ was firstly mixed with $\mathrm{Hg}^{2+}(25$ $\mu \mathrm{M})$ and incubated for $5 \mathrm{~min}$. Then, ${ }_{\mathrm{L}}$-Cys was added at different concentrations $(0.1-30.0 \mu \mathrm{M})$. After the mixed solution was incubated at room temperature for $5 \mathrm{~min}$, the FL intensity was recorded (excited at $370 \mathrm{~nm}$ ). The relative fluorescence recovery ratio $\left(F_{1}-F\right) / F_{1}$ was used to evaluate the fluorescence recovery by L-Cys, where $F$ and $F_{1}$ represent the FL intensity in absence and presence of $\mathrm{L}_{\mathrm{L}} \mathrm{Cys}$, respectively.

\section{Results and discussion}

\subsection{Gram-scale synthesis of N-GQDs}

In present work, N-GQDs were synthesized using one-step hydrothermal fusion between trinitropyrene and tryptophan in $\mathrm{NaOH}$ medium (Fig. 1). Trinitropyrene was chosen as precursor because it has the same mother-nucleus structure as graphene and could be fused at alkaline medium. Tryptophan with $\mathrm{N}$ atoms and carboxyl groups was applied as dopant to introduce doped heteroatoms as well as functional groups on GQDs. As demonstrated in inset of Fig. 2A, GQDs with blue fluorescence are obtained. In order to obtain GQDs with excellent fluorescent properties, the conditions of hydrothermal reaction were optimized. The highest fluorescence (FL) intensity is obtained at a reaction of $6 \mathrm{~h}$ and $200{ }^{\circ} \mathrm{C}$ (Fig. S1A and S1B $\dagger$ ). Obviously, high temperature can facilitate the efficiency of the bottom-up fusion. However, higher temperature was not 

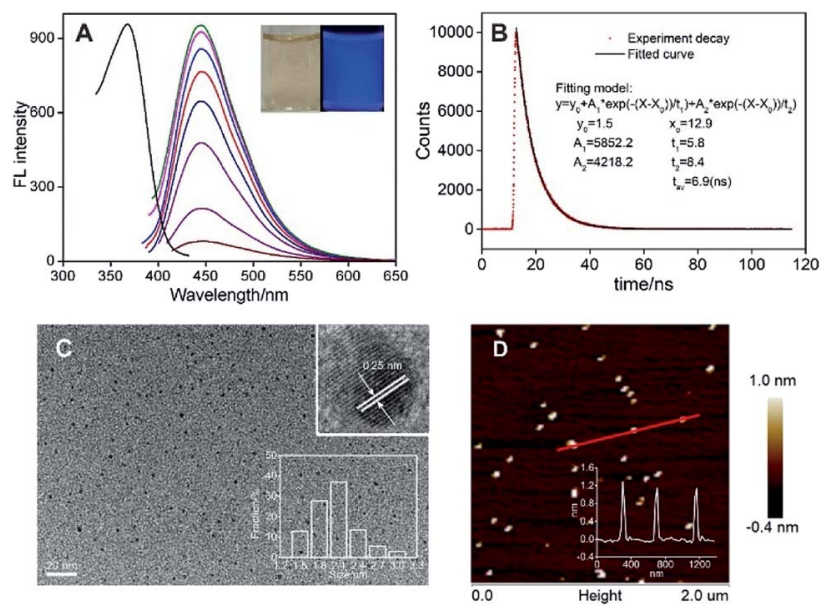

Fig. 2 (A) The FL excitation spectrum (EX, left) obtained at the emission wavelength of $445 \mathrm{~nm}$ and $\mathrm{FL}$ emission spectra (EM, right) of $\mathrm{N}$ GQDs obtained with different excitation wavelength (340 to $410 \mathrm{~nm}$, $10 \mathrm{~nm}$ increment). Insets are N-GQDs solution under daylight (left) and UV light (365 nm). (B) FL lifetime spectrum of N-GQDs. (C) TEM images of N-GQDs. Insets were the high-resolution TEM (HRTEM) image with indicated lattice parameter and size distribution of N-GQDs. (D) AFM image of the N-GQDs. Inset was the height profile along the red line.

investigated because it would have exceeded the tolerated range of the common Teflon-lined autoclave. Too short reaction time results in low concentration of GQDs due to incomplete molecule fusion, whereas too long time might result in GQDs stack. The FL intensity increases with the increase of tryptophan concentration and then reaches a plateau, presumably because the fusion between trinitropyrene and tryptophan is saturated (Fig. S1C $\dagger$ ). The concentration of $\mathrm{NaOH}$ exhibits similar effects on the FL intensity of GQDs (Fig. S1D $\dagger$ ). Thus, N-GQDs synthesized under the optimized conditions $(75 \mathrm{mM}$ L-tryptophan and $125 \mathrm{mM} \mathrm{NaOH}$ at $200{ }^{\circ} \mathrm{C}$ for $6 \mathrm{~h}$ ) was applied for further investigation.

The synthesis yield of obtained N-GQDs is as high as $91.2 \%$, indicating high efficiency for the preparation of N-GQDs. It is worth noting that the volume of the autoclave in present work is $500 \mathrm{~mL}$, which is significantly higher than that used in most GQDs synthesis (usually $25 \mathrm{~mL}$ or $50 \mathrm{~mL}$ volume). Even the reaction solution is only $40 \%$ of the volume of autoclave, more than $0.5 \mathrm{~g}$ of N-GQDs could be produced at a single run. Thus, gram-scale synthesis of N-GQDs could be realized using this green and one-step strategy.

\subsection{Fluorescence property, morphology and composition of N-GQDs}

Fig. 2A demonstrates the fluorescent excitation and emission spectra of N-GQDs. As shown, the maximum excitation at $370 \mathrm{~nm}$ gives the maximum emission at $445 \mathrm{~nm}$. It is worth noting that the maximum FL emission of N-GQDs remains unchanged when the excitation wavelength changes. In addition, a narrow emission is revealed with the half-peak width of about $55 \mathrm{~nm}$ (excited at $370 \mathrm{~nm}$ ). These emission-independent and narrow emission suggest that N-GQDs have uniform structure and luminescent states. The absolute quantum yield is $24.8 \%$ with a lifetime of 6.9 ns (Fig. 2B).

As revealed by transmission electron microscopy (TEM), the size of N-GQDs is uniform with average size of $\sim 2.1 \mathrm{~nm}$. The crystallinity of N-GQDs was confirmed by high resolution TEM (HRTEM). The lattice spacing of $0.25 \mathrm{~nm}$ corresponds to the graphene (100) planes (Fig. 2C). Good crystallinity of N-GQDs might be attributed to the trinitropyrene precursor (Fig. 1), which has the mother-nucleus structure as graphene. The atomic force microscopic (AFM) image shows that the thickness of N-GQDs is about $1.0 \mathrm{~nm}$. Considering the doped heteroatoms and surface groups, the as-prepared N-GQDs are mostly singlelayered (Fig. 2D). The uniform size, good crystalline and homogeneous thickness suggest excellent structure characteristics of N-GQDs, which also support the narrow and emissionindependent fluorescence.

The composition of N-GQDs was investigated by X-ray photoelectron spectroscopy (XPS). XPS survey spectrum reveals the presence of $\mathrm{C}, \mathrm{O}$, and $\mathrm{N}$ with atomic percentages of $78.7 \%, 17.3 \%$ and $4.0 \%$, respectively (Fig. 3A), indicating successful introduction of $\mathrm{N}$ atoms on GQDs. The high resolution $\mathrm{C} 1 \mathrm{~s}$ spectrum clearly evidences the presence of $\mathrm{C}-\mathrm{C}=\mathrm{C}$ $\left(\mathrm{sp}^{2} \mathrm{C}, 284.6 \mathrm{eV}\right), \mathrm{C}-\mathrm{O} / \mathrm{C}-\mathrm{N}(285.5 \mathrm{eV})$ and $\mathrm{O}-\mathrm{C}=\mathrm{O}(288.0 \mathrm{eV})$, respectively (Fig. 3B). ${ }^{17}$ According to the high resolution $\mathrm{O} 1 \mathrm{~s}$ spectrum, N-GQDs contains carboxyl group (532.8 eV) and hydroxyl group (531.4 eV) (Fig. 3C). ${ }^{33}$ The pyrrole N (C-N-C, $398.7 \mathrm{eV})$ and graphite $\mathrm{N}\left(\mathrm{N}-(\mathrm{C})_{3}, 399.2 \mathrm{eV}\right)$ in high resolution $\mathrm{N}$ 1s spectrum indicate that $\mathrm{N}$ atoms have successfully doped into the carbon skeleton of GQDs. ${ }^{34}$ In addition, N-GQDs also contain some primary amino groups $(\mathrm{C}-\mathrm{N}-\mathrm{H}, 400.1 \mathrm{eV})$ (Fig. 3D).

\subsection{Selective fluorescence quenching of N-GQDs towards $\mathrm{Hg}^{2+}$}

The selectivity of N-GQDs towards different metal ions was investigated. As demonstrated in Fig. $4 \mathrm{~A}, \mathrm{~K}^{+}, \mathrm{Na}^{+}, \mathrm{Ca}^{2+}, \mathrm{Mg}^{2+}$,
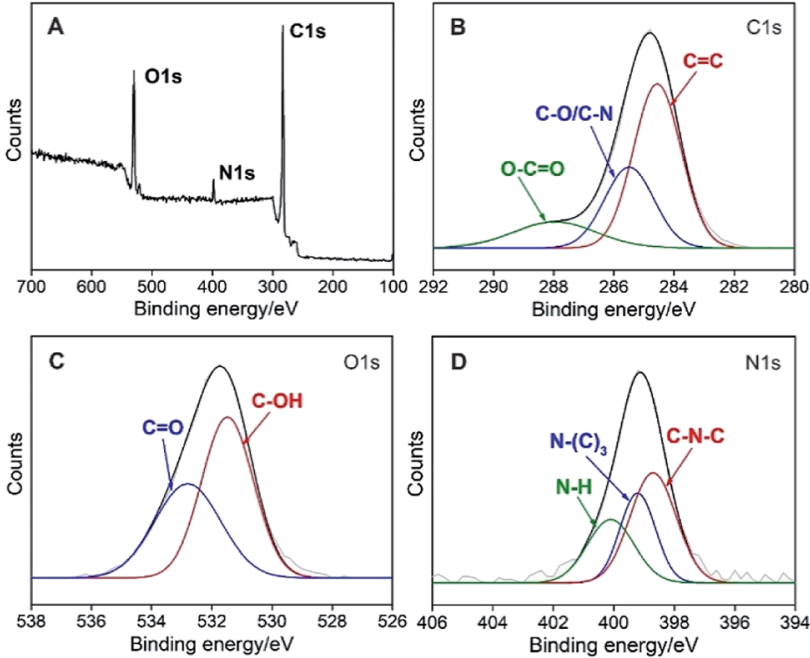

Fig. 3 XPS survey spectrum (A) and high resolution $C$ is (B), O 1s (C), and $\mathrm{N}$ 1s (D) spectra of $\mathrm{N}-\mathrm{GQDs}$. 

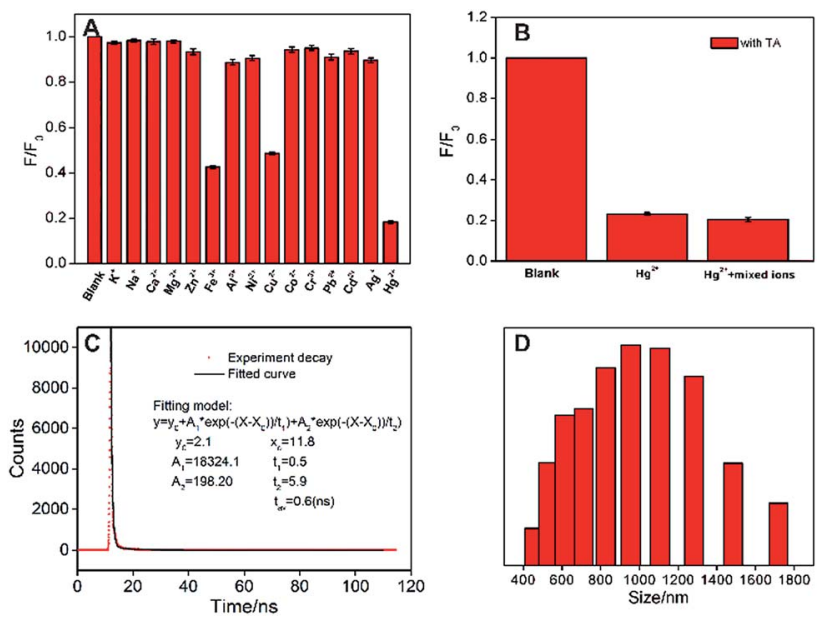

Fig. 4 (A) The relative FL ratio of the N-GQDs in the presence of different metal ions $(25 \mu \mathrm{M})$. (B) The relative FL ratio of the $N-G Q D$ s in the presence of $\mathrm{Hg}^{2+}$ or $\mathrm{Hg}^{2+}+$ mixed ions (all other ions in $\mathrm{A}$ ) with tartaric acid (TA, $125 \mu \mathrm{M})$. (C) FL lifetime spectrum of N-GQDs in the presence of $\mathrm{Hg}^{2+}$. (D) The dynamic light scattering of $\mathrm{N}-\mathrm{GQDs}$ in the presence of $\mathrm{Hg}^{2+}$.

$\mathrm{Zn}^{2+}, \mathrm{Al}^{3+}, \mathrm{Ni}^{2+}, \mathrm{Co}^{2+}, \mathrm{Cr}^{3+}, \mathrm{Pb}^{2+}, \mathrm{Cd}^{2+}$, and $\mathrm{Ag}^{+}$hardly affect the fluorescence of N-GQDs. On the contrary, $\mathrm{Hg}^{2+}, \mathrm{Fe}^{3+}$ and $\mathrm{Cu}^{2+}$ could quench the fluorescence of N-GQDs. However, the quenching efficiency of $\mathrm{Hg}^{2+}$ is significantly higher than that of $\mathrm{Fe}^{3+}$ and $\mathrm{Cu}^{2+}$, indicating the potential for sensitive detection of $\mathrm{Hg}^{2+}$. In contrast to most reported GQDs-based fluorescent sensors, which usually applied to detect $\mathrm{Fe}^{3+} \mathrm{Or} \mathrm{Cu}^{2+}$, GQDs with $\mathrm{Hg}^{2+}$ sensitivity is rarely reported. Tartaric acid (TA) is chosen as masking agent to eliminate the interference of $\mathrm{Fe}^{3+}$ and $\mathrm{Cu}^{2+}$. As shown in Fig. S2, $\uparrow$ the addition of TA has no effect on the fluorescence of N-GQDs. In the presence of TA $(125 \mu \mathrm{M})$, the fluorescence of N-GQDs quenched by $\mathrm{Hg}^{2+}$ is similar with that obtained in mixture of $\mathrm{Hg}^{2+}$ and all other ions in Fig. 4A (Fig. 4B). Hence, selective fluorescence response of N-GQDs towards $\mathrm{Hg}^{2+}$ could be achieved.

The mechanism for the fluorescence quenching of N-GQDs by $\mathrm{Hg}^{2+}$ was investigated. Firstly, we measured the fluorescence lifetime of N-GQDs after interaction with $\mathrm{Hg}^{2+}$. The lifetime of N-GQDs in presence of $\mathrm{Hg}^{2+}$ is $0.6 \mathrm{~ns}$ (Fig. 4C), which is remarkably shorter than that of N-GQDs itself $(6.9 \mathrm{~ns})$. This indicates fluorescence quenching by electron transfer between $\mathrm{N}-\mathrm{GQDS}$ and $\mathrm{Hg}^{2+}$, presumably because of the electron-donating moieties on GQDs formed by doped $\mathrm{N}$ atoms (Fig. 1). In addition, the remarkable change of particle size was also investigated by the dynamic light scattering (DLS). The average size of particles in N-GQDs- $\mathrm{Hg}^{2+}$ mixture is $1038.7 \mathrm{~nm}$, indicating $\mathrm{Hg}^{2+}$-induced aggregation of N-GQDs. (Fig. 4D). Thus, aggregation-induced-quenching also occurs (Fig. 1).

\subsection{Fluorescent turn-off detection of $\mathrm{Hg}^{2+}$ using N-GQDs as fluorescent probes}

Owning to selective fluorescence quenching by $\mathrm{Hg}^{2+}$, fluorescent sensor (turn-off mode) was developed for the detection of $\mathrm{Hg}^{2+}$ using N-GQDs as fluorescent probe. To achieve the highest
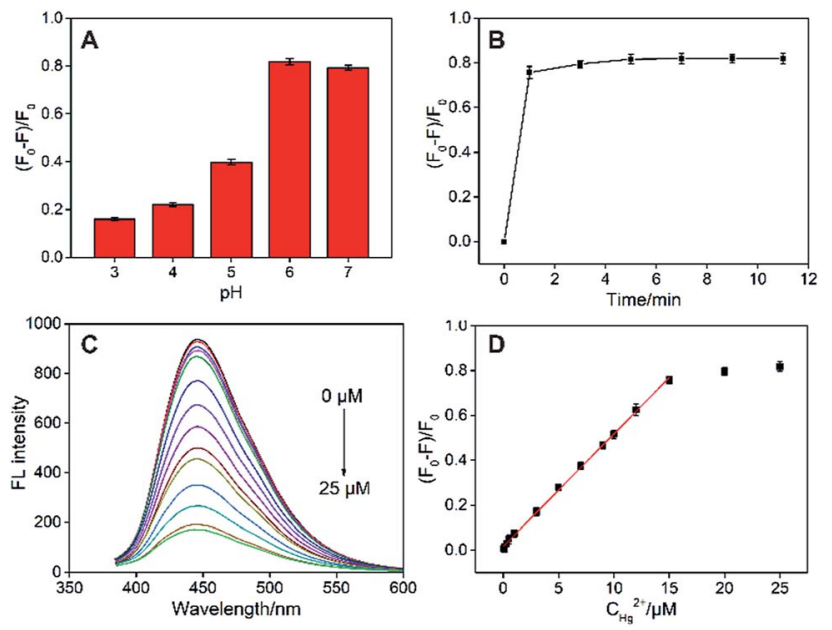

Fig. 5 (A) The relative FL ratio of $\mathrm{N}-\mathrm{GQDs}$ with $\mathrm{Hg}^{2+}$ at different $\mathrm{pH}$. (B) The effect of incubation time on fluorescent quenching ratio of $\mathrm{N}$ GQDs caused by $\mathrm{Hg}^{2+}(25 \mu \mathrm{M})$. (C) FL emission spectra of $\mathrm{N}-\mathrm{GQDs}$ in the presence of different concentrations of $\mathrm{Hg}^{2+}$. (D) The linear dependence of $\left(F_{0}-F\right) / F_{0}$ on concentration of $\mathrm{Hg}^{2+}$

sensitivity, the detection conditions including $\mathrm{pH}$ and incubation time were optimized. As shown in Fig. 5A, the highest fluorescence quenching exists at $\mathrm{pH}$. Too low a $\mathrm{pH}$ value $\mathrm{pH}$ 3-5) might weaken the interaction between N-GQDs and metal ions through protonation of $\mathrm{N}$ atoms on GQDs whereas too high a $\mathrm{pH}$ value $(\mathrm{pH} 7)$ possibly converts metal ions into hydroxide. At the same time, fast kinetics is observed and a plateau of fluorescence intensity appeared within 3 min (Fig. 5B), indicating the strong interaction between N-GQDs and $\mathrm{Hg}^{2+}$. Under the optimized conditions, remarkable decrease in FL intensity towards different concentration of N-GQDs is observed (Fig. 5C). Good linearity existed between fluorescent quenching ratio $\left(F_{0}-F\right) / F_{0}$ and $\mathrm{Hg}^{2+}$ concentration from $50 \mathrm{nM}$ to $15 \mu \mathrm{M}$ (Fig. 5D). The limit of detection (LOD) is $19 \mathrm{nM}$ at a signal-tonoise ratio of 3. As shown in Table S1 (ESI $\dagger$ ), the detection limit obtained with the present method was lower than those obtained by carbon dots (CDs), ${ }^{35} \mathrm{~F}$ doped CDs (F-CDs), ${ }^{36} \mathrm{~N}$ doped CDs (N-CDs), ${ }^{37} \mathrm{~N}$ and $\mathrm{S}$ co-doped CDs (N, S-CDs), ${ }^{38,39} \mathrm{~N}$ doped GQDs (N-GCDs), ${ }^{34}$ sulfur and nitrogen co-doped GQDs (S,N-GQDs), ${ }^{40}$ and N-GQDs, ${ }^{41}$ but higher than that obtained using Rhodamine B assisted GQDs (RhB-GQDs) ${ }^{42}$ and $\mathrm{N}$ and $\mathrm{S}$ co-doped GQDs (N, S-GQDs). ${ }^{43}$

The application of the as-prepared fluorescent sensor for detection of $\mathrm{Hg}^{2+}$ in environmental samples (lake water) was

Table 1 Detection of $\mathrm{Hg}^{2+}$ in environmental water samples

Concentration of $(\mu \mathrm{M})$

Sample Added $\mathrm{Hg}^{2+}$ Found by N-GQDs $\operatorname{RSD}(n=3, \%) \quad(\%)$

$\begin{array}{ccccc}1 & 3.00 & 2.91,2.83,2.96 & 2.2 & 96.7 \\ 2 & 7.00 & 6.72,6.65,6.88 & 1.8 & 96.4 \\ 3 & 12.0 & 11.6,11.9,12.0 & 1.7 & 98.6\end{array}$


investigated (Table 1). As the original $\mathrm{Hg}^{2+}$ is not detected, the standard addition method was applied to evaluate the detection reliability. Good recoveries ranged from $96.4 \%$ to $98.6 \%$ were obtained, indicating the potential application of N-GQDs in real analysis.

\subsection{Detection of L-Cys based on fluorescent off-on mode}

As illustrated in Fig. 6A, the fluorescence of N-GQDs quenched by $\mathrm{Hg}^{2+}$ could be recovered after addition of $\mathrm{L}$-Cys to the $\mathrm{N}$ GQDs- $\mathrm{Hg}^{2+}$ system. As shown in Fig. 6B, L-Cys itself has no effect on the fluorescence intensity of N-GQDs. The possible interference of other nine common amino acids on the detection of L-Cys was investigated. As shown in Fig. 6A, these amino acids could not significantly restore the fluorescence of N-GQDs quenched by $\mathrm{Hg}^{2+}$, indicating good selectivity of L-Cys. We speculate that the fluorescence recovery by L-Cys is due to the specific binding between $-\mathrm{SH}$ and $\mathrm{Hg}^{2+}$. In support of this notion, glutathione (GSH, tripeptide composed of Cys, glycine, and glutamic acid) and homocysteine (Hcy, only include an additional $-\mathrm{CH}_{2}-$ before $-\mathrm{SH}$ in comparison with Cys) also recover the fluorescence to some extent because of the similar structure as Cys (Fig. S3†). Fluorescence turn-off-on sensing mode could be developed for individual detection of L-Cys as a proof-of-demonstration. As shown in Fig. 6C, the fluorescence of N-GQDs gradually restores with increasing the concentration of L-Cys. Good linear relationship is found between fluorescence recovery rate and the concentration of $\mathrm{L}_{\mathrm{L}}$-Cys from $100 \mathrm{nM}$ to 50 $\mu \mathrm{M}$ (DL of $29 \mathrm{nM}$ ) (Fig. 6D). As shown in Table S2, $\dagger$ the detection limit obtained with the present method was lower than those obtained by N,S-CDs-Fe ${ }^{3+},{ }^{44} \mathrm{~N}-\mathrm{CDs}-\mathrm{Hg}^{2+},{ }^{47} \mathrm{Eu}-\mathrm{GQDS}-\mathrm{Cu}^{2+},{ }^{48}$ and $\mathrm{N}-\mathrm{GQDS}-\mathrm{Hg}^{2+}{ }^{49}$ but higher than that obtained using CDs$\mathrm{Hg}^{2+},{ }^{45} \mathrm{~N}-\mathrm{CDs} @ \mathrm{~V}_{2} \mathrm{O}_{5},{ }^{46}$ and N-GQDs- $\mathrm{Hg}^{2+} \cdot{ }^{49}$ The application of the N-GQDs- $\mathrm{Hg}^{2+}$ system for detection of $\mathrm{L}-\mathrm{Cys}$ in environmental
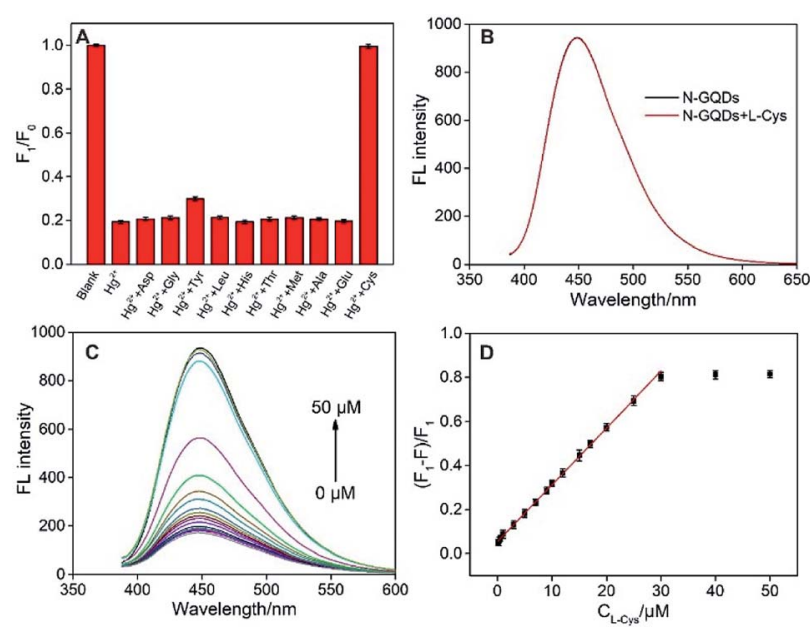

Fig. 6 (A) The relative FL ratio of $\mathrm{N}-\mathrm{GQDs}$ with $\mathrm{Hg}^{2+}$ in the presence of different amino acid. (B) $\mathrm{FL}$ emission spectra of $\mathrm{N}$-GQDs in the absence and presence of L-Cys $(50 \mu \mathrm{M})$. (C) FL emission spectra of $\mathrm{N}$ GQDs with $\mathrm{Hg}^{2+}(25 \mu \mathrm{M})$ in the presence of different concentrations of $L-C y s$. (D) The linear dependence of $\left(F_{1}-F\right) / F_{1}$ on concentration of $L-$ Cys. samples (lake water) was investigated using standard addition method (Table S3†). Good recoveries ranged from $94.1 \%$ to $97.8 \%$ were obtained.

\section{Conclusions}

Gram-scale synthesis of nitrogen doped graphene quantum dots (N-GQDs) with $\mathrm{Hg}^{2+}$ sensitivity was readily achieved using one-step hydrothermal process. Such blue N-GQDs exhibit good characteristics including uniform size, good crystalline, bright and emission-independent fluorescence and single-layered graphene thickness. The ability to interact with $\mathrm{Hg}^{2+}$ results in selective and sensitive fluorescence quenching through electron transfer and aggregation. With N-GQDs being fluorescent probes, turn-off sensing of $\mathrm{Hg}^{2+}$ and turn-off-on detection of L-cysteine were demonstrated. The easy preparation and good characteristics of N-GQDs promise a large variety of applications, including optical sensing, photocatalysis and bioimaging.

\section{Conflicts of interest}

There are no conflicts to declare.

\section{Acknowledgements}

The authors gratefully acknowledge the financial support from the National Natural Science Foundation of China (No. 81860512), Guangxi Natural Science Foundation (2016GXNSFBA380194), and the Zhejiang Provincial Natural Science Foundation of China (LY19B050008).

\section{References}

1 H. N. Kim, W. X. Ren, J. S. Kim and J. Yoon, Fluorescent and colorimetric sensors for detection of lead, cadmium, and mercury ions, Chem. Soc. Rev., 2012, 41, 3210-3244.

2 M. Gao, X. Lu, S. Chen, D. Tian, Y. Zhu and C. Wang, Enhanced peroxidase-like activity of $\mathrm{Mo}^{6+}$-doped $\mathrm{Co}_{3} \mathrm{O}_{4}$ nanotubes for ultrasensitive and colorimetric L-cysteine detection, ACS Appl. Nano Mater., 2018, 1, 4703-4715.

3 Y. Liu, Y. Deng, T. Li, Z. Chen, H. Chen, S. Li and H. Liu, Aptamer-based electrochemical biosensor for mercury ions detection using AuNPs-modified glass carbon electrode, $J$. Biomed. Nanotechnol., 2018, 14, 2156-2161.

4 S. Y. Ding, M. Dong, Y. W. Wang, Y. T. Chen, H. Z. Wang, C. Y. Su and W. Wang, Thioether-based fluorescent covalent organic framework for selective detection and facile removal of mercury(II), J. Am. Chem. Soc., 2016, 138, 3031-3037.

5 G. H. Chen, W. Y. Chen, Y. C. Yen, C. W. Wang, H. T. Chang and C. F. Chen, Detection of mercury(II) ions using colorimetric gold nanoparticles on paper-based analytical devices, Anal. Chem., 2014, 86, 6843-6849.

6 M. Gao, X. Lu, M. Chi, S. Chen and C. Wang, Fabrication of oxidase-like hollow $\mathrm{MnCo}_{2} \mathrm{O}_{4}$ nanofibers and their sensitive 
colorimetric detection of sulfite and L-cysteine, Inorg. Chem. Front., 2017, 4, 1862-1869.

7 H. Li, L. Ye, Y. Wang and C. Xie, A glassy carbon electrode modified with hollow cubic cuprous oxide for voltammetric sensing of L-cysteine, Microchim. Acta, 2018, 185, 5.

8 X. Li, X. Y. Yang, J. Q. Sha, T. Han, C. J. Du, Y. J. Sun and Y. Q. Lan, POMOF/SWNT nanocomposites with prominent peroxidase-mimicking activity for L-cysteine "on-off switch" colorimetric biosensing, ACS Appl. Mater. Interfaces, 2019, 11, 16896-16904.

9 Y. C. Liang, Q. Zhao, X. Y. Wu, Z. Li, Y. J. Lu, Q. Liu, L. Dong and C. X. Shan, A ratiometric fluorescent nanoprobe based on quenched carbon dots-rhodamine $\mathrm{B}$ for selective detection of L-cysteine, J. Alloys Compd., 2019, 788, 615-622.

10 Y. Zhu, Z. Xu, K. Yan, H. Zhao and J. Zhang, One-Step Synthesis of $\mathrm{CuO}-\mathrm{Cu}_{2} \mathrm{O}$ heterojunction by flame spray pyrolysis for cathodic photoelectrochemical sensing of $\mathrm{L}^{-}$ cysteine, ACS Appl. Mater. Interfaces, 2017, 9, 40452-40460.

11 J. Wu, P. Ran, S. Zhu, F. Mo, C. Wang and Y. Fu, A highly sensitive electrochemiluminescence sensor for the detection of L-cysteine based on the rhombus-shaped rubrene microsheets and platinum nanoparticles, Sens. Actuators, B, 2019, 278, 97-102.

12 Y. Guo, Z. Wang, H. Shao and X. Jiang, Hydrothermal synthesis of highly fluorescent carbon nanoparticles from sodium citrate and their use for the detection of mercury ions, Carbon, 2013, 52, 583-589.

13 P. Makam, R. Shilpa, A. E. Kandjani, S. R. Periasamy, Y. M. Sabri, C. Madhu, S. K. Bhargava and T. Govindaraju, SERS and fluorescence-based ultrasensitive detection of mercury in water, Biosens. Bioelectron., 2018, 100, 556-564.

14 F. Yan, Y. Zou, M. Wang, X. Mu, N. Yang and L. Chen, Highly photoluminescent carbon dots-based fluorescent chemosensors for sensitive and selective detection of mercury ions and application of imaging in living cells, Sens. Actuators, B, 2014, 192, 488-495.

15 Y. Yan, J. Gong, J. Chen, Z. Zeng, W. Huang, K. Pu, J. Liu and P. Chen, Recent advances on graphene quantum dots: From chemistry and physics to applications, Adv. Mater., 2019, 31, e1808283.

16 Y. Du and S. Guo, Chemically doped fluorescent carbon and graphene quantum dots for bioimaging, sensor, catalytic and photoelectronic applications, Nanoscale, 2016, 8, 25322543.

17 L. Wang, Y. Wang, T. Xu, H. Liao, C. Yao, Y. Liu, Z. Li, Z. Chen, D. Pan, L. Sun and M. Wu, Gram-scale synthesis of single-crystalline graphene quantum dots with superior optical properties, Nat. Commun., 2014, 5, 5357.

18 S. Zhu, Y. Song, J. Wang, H. Wan, Y. Zhang, Y. Ning and B. Yang, Photoluminescence mechanism in graphene quantum dots: Quantum confinement effect and surface/ edge state, Nano Today, 2017, 13, 10-14.

19 X. Yao, X. Niu, K. Ma, P. Huang, J. Grothe, S. Kaskel and Y. Zhu, Graphene quantum dots-capped magnetic mesoporous silica nanoparticles as a multifunctional platform for controlled drug delivery, magnetic hyperthermia, and photothermal therapy, Small, 2017, 13, 1602225.

20 J. Dong, K. Wang, L. Sun, B. Sun, M. Yang, H. Chen, Y. Wang, J. Sun and L. Dong, Application of graphene quantum dots for simultaneous fluorescence imaging and tumor-targeted drug delivery, Sens. Actuators, B, 2018, 256, 616-623.

21 Y. Yan, J. Chen, N. Li, J. Tian, K. Li, J. Jiang, J. Liu, Q. Tian and P. Chen, Systematic bandgap engineering of graphene quantum dots and applications for photocatalytic water splitting and $\mathrm{CO}_{2}$ reduction, ACS Nano, 2018, 12, 3523-3532.

22 J. Tian, J. Chen, J. Liu, Q. Tian and P. Chen, Graphene quantum dot engineered nickel-cobalt phosphide as highly efficient bifunctional catalyst for overall water splitting, Nano Energy, 2018, 48, 284-291.

23 F. Xi, J. Zhao, C. Shen, J. He, J. Chen, Y. Yan, K. Li, J. Liu and P. Chen, Amphiphilic graphene quantum dots as a new class of surfactants, Carbon, 2019, 153, 127-135.

24 Z. Zeng, F. Xiao, H. Phan, S. Chen, Z. Yu, R. Wang, T. Nguyen and T. Tan, Unraveling the cooperative synergy of zerodimensional graphene quantum dots and metal nanocrystals enabled by layer-by-layer assembly, J. Mater. Chem. A, 2018, 6, 1700-1713.

25 Q. Wang, Z. Jin, D. Chen, D. Bai, H. Bian, J. Sun, G. Zhu, G. Wang and S. F. Liu, $\mu$-Graphene crosslinked $\mathrm{CsPbI}_{3}$ quantum dots for high efficiency solar cells with much improved stability, Adv. Energy Mater., 2018, 8, 1800007.

26 C. Shen, S. Ge, Y. Pang, F. Xi, J. Liu, X. Dong and P. Chen, Facile and scalable preparation of highly luminescent N,S co-doped graphene quantum dots and their application for parallel detection of multiple metal ions, J. Mater. Chem. B, 2017, 5, 6593-6600.

27 S. Bian, C. Shen, Y. Qian, J. Liu, F. Xi and X. Dong, Facile synthesis of sulfur-doped graphene quantum dots as fluorescent sensing probes for $\mathrm{Ag}^{+}$ions detection, Sens. Actuators, B, 2017, 242, 231-237.

28 D. Chen, X. Zhuang, J. Zhai, Y. Zheng, H. Lu and L. Chen, Preparation of highly sensitive $\mathrm{Pt}$ nanoparticles-carbon quantum dots/ionic liquid functionalized graphene oxide nanocomposites and application for $\mathrm{H}_{2} \mathrm{O}_{2}$ detection, Sens. Actuators, B, 2018, 255, 1500-1506.

29 B. Y. Fang, C. Li, Y. Y. Song, F. Tan, Y. C. Cao and Y. D. Zhao, Nitrogen-doped graphene quantum dot for direct fluorescence detection of $\mathrm{Al}^{3+}$ in aqueous media and living cells, Biosens. Bioelectron., 2018, 100, 41-48.

30 S. Bian, C. Shen, H. Hua, L. Zhou, H. Zhu, F. Xi, J. Liu and X. Dong, One-pot synthesis of sulfur-doped graphene quantum dots as a novel fluorescent probe for highly selective and sensitive detection of lead(II), RSC Adv., 2016, 6, 69977-69983.

31 L. Lu, L. Zhou, J. Chen, F. Yan, J. Liu, X. Dong, F. Xi and P. Chen, Nanochannel confined graphene quantum dots for ultrasensitive electrochemical analysis of complex samples, ACS Nano, 2018, 12, 12673-12681.

$32 \mathrm{~J}$. Ju, R. Zhang and W. Chen, Photochemical deposition of surface-clean silver nanoparticles on nitrogen-doped graphene quantum dots for sensitive colorimetric detection of glutathione, Sens. Actuators, B, 2016, 228, 66-73. 
33 Q. Xiao, S. Lu, C. Huang, W. Su and S. Huang, Novel N-doped carbon dots/beta-cyclodextrin nanocomposites for enantioselective recognition of tryptophan enantiomers, Sensors, 2016, 16, 1874.

34 Z. Yan, X. Qu, Q. Niu, C. Tian, C. Fan and B. Ye, A green synthesis of highly fluorescent nitrogen-doped graphene quantum dots for the highly sensitive and selective detection of mercury(II) ions and biothiols, Anal. Methods, 2016, 8, 1565-1571.

35 H. Goncalves, A. Duarte and J. Silva, Optical fiber sensor for $\mathrm{Hg}$ (II) based on carbon dots, Biosens. Bioelectron., 2010, 26, 1302-1306.

36 Z. Gao, Z. Lin, X. Chen, Z. Lai and Z. Huang, Carbon dotsbased fluorescent probe for trace $\mathrm{Hg}^{2+}$ detection in water sample, Sens. Actuators, B, 2016, 222, 965-971.

37 R. Zhang and W. Chen, Nitrogen-doped carbon quantum dots: Facile synthesis and application as a "turn-off" fluorescent probe for detection of $\mathrm{Hg}^{2+}$ ions, Biosens. Bioelectron., 2014, 55, 83-90.

38 Y. Wang, S. Kim and L. Feng, Highly luminescent N, S-codoped carbon dots and their direct use as mercury (II) sensor, Anal. Chim. Acta, 2015, 890, 134-142.

39 L. Li, B. Yu and T. You, Nitrogen and sulfur co-doped carbon dots for highly selective and sensitive detection of $\mathrm{Hg}$ (II) ions, Biosens. Bioelectron., 2015, 74, 263-269.

40 E. Sharma, D. Vashisht, A. Vashisht, V. Vats, S. Mehta and K. Singh, Facile synthesis of sulfur and nitrogen codoped graphene quantum dots for optical sensing of $\mathrm{Hg}$ and $\mathrm{Ag}$ ions, Chem. Phys. Lett., 2014, 55, 83-90.

41 Y. Liu, X. Tang, M. Deng, Y. Cao, Y. Li, H. Zhang, F. Li, F. Yan, T. Lan, L. Shi, L. Gao, L. Huang, T. Zhu, H. Lin, Y. Bai, D. Qu, X. Huang and F. Qiu, Nitrogen doped graphene quantum dots as a fluorescent probe for mercury(II) ions, Microchim. Acta, 2019, 186, 140-147.
42 Y. Yang, X. Xiao, X. Xing, Z. Wang, T. Zou, Z. Wang, R. Zhao and $\mathrm{Y}$. Wang, Rhodamine $\mathrm{B}$ assisted graphene quantum dots flourescent sensor system for sensitive recognition of mercury ions, J. Lumin., 2019, 207, 273-281.

43 N. Anh, A. Chowdhury and R. Doong, Highly sensitive and selective detection of mercury ions using $\mathrm{N}$, S-codoped graphene quantum dots and its paper strip based sensing application in wastewater, Sens. Actuators, B, 2017, 252, 1169-1178.

$44 \mathrm{H}$. Wu, J. Jiang, X. Gu and C. Tong, Nitrogen and sulfur codoped carbon quantum dots for highly selective and sensitive fluorescent detection of $\mathrm{Fe}$ (III) ions and $\mathrm{L}^{-}$ cysteine, Microchim. Acta, 2017, 184(7), 2291-2298.

45 F. Yan, D. Shi, T. Zheng, K. Yun and X. Zhou, Carbon dots as nanosensor for sensitive and selective detection of $\mathrm{Hg}^{2+}$ and L-Cysteine by means of fluorescence "Off-On" switching, Sens. Actuators, B, 2016, 224, 926-935.

46 A. B. Ganganboina, A. C. Dutta and R. A. Doong, N-doped graphene quantum dots-decorated $\mathrm{V}_{2} \mathrm{O}_{5}$ nanosheet for fluorescence turn off-on detection of cysteine, ACS Appl. Mater. Interfaces, 2018, 10, 614-624.

47 H. Huang, Y. Weng, L. Zheng, B. Yao, W. Weng and X. Lin, Nitrogen-doped carbon quantum dots as fluorescent probe for "off-on" detection of mercury ions, L-cysteine and iodide ions, J. Colloid Interface Sci., 2017, 506, 373-378.

48 L. Lin, X. Song, Y. Chen, M. Rong, Y. Wang, L. Zhao, T. Zhao and $\mathrm{X}$. Chen, Europium-decorated graphene quantum dots as a fluorescent probe for label-free, rapid and sensitive detection of $\mathrm{Cu}^{2+}$ and L-cysteine, Anal. Chim. Acta, 2015, 891, 261-268.

49 Z. Liu, Y. Gong and Z. Fan, Cysteine detection using a highfluorescence sensor based on a nitrogen-doped graphene quantum dot-mercury(II) system, J. Lumin., 2016, 175, 129134. 